Revue internationale P.M.E.

Économie et gestion de la petite et moyenne entreprise

\title{
Profils de chercheurs primo-entrepreneurs et stratégies de croissance de la jeune entreprise innovante
}

\author{
Jean-Pierre Boissin, Marie-Christine Chalus-Sauvannet, Bérangère Deschamps \\ et Sébastien Geindre
}

Volume 22, numéro 2, 2009

URI : https://id.erudit.org/iderudit/044030ar

DOI : https://doi.org/10.7202/044030ar

Aller au sommaire du numéro

Éditeur(s)

Presses de l'Université du Québec

ISSN

0776-5436 (imprimé)

1918-9699 (numérique)

Découvrir la revue

Citer cet article

Boissin, J.-P., Chalus-Sauvannet, M.-C., Deschamps, B. \& Geindre, S. (2009). Profils de chercheurs primo-entrepreneurs et stratégies de croissance de la jeune entreprise innovante. Revue internationale P.M.E., 22(2), 41-65.

https://doi.org/10.7202/044030ar
Résumé de l'article

À travers l'étude de quatre entreprises dans le secteur de la haute technologie, les auteurs s'intéressent au rôle joué par le dirigeant sur la stratégie de croissance. Une attention particulière est portée sur le profil du chercheur primo-entrepreneur.

Dans un premier temps, les critères de mesure de la croissance sont discutés et mis en relation avec le lien entre dirigeant et croissance; dans un deuxième temps, la méthodologie et les quatre cas étudiés sont présentés. Enfin, les résultats sont exposés en soulignant les liens entre profil de dirigeant et croissance. 


\title{
Profils de chercheurs primo-entrepreneurs et stratégies de croissance de la jeune entreprise innovante
}

\author{
Jean-Pierre BOISSIN \\ Marie-Christine CHALUS-SAUVANNET \\ Université Pierre-Mendès-France Grenoble II \\ Bérangère DESCHAMPS \\ Sébastien GEINDRE \\ IAE de Grenoble
}

MOTS CLÉS

\section{Dirigeant - Primo-entrepreneur - Chercheur - Croissance Jeune entreprise innovante - Haute technologie}

\begin{abstract}
LES AUTEURS
JEAN-PIERRE BOISSIN est professeur des universités à l'Université Pierre-Mendès-France, IAE de Grenoble. II est directeur du laboratoire CERAG UMR CNRS-UPMF. Ses travaux portent sur la gouvernance et les stratégies de croissance des entreprises innovantes, l'intention entrepreneuriale et la structuration de la recherche à partir d'outils bibliométriques. II a fondé la première Maison de l'entrepreneuriat à Grenoble en 2002.

MARIE-Christine Chalus-SAUVANNET est maître de conférences à l'Université de Grenoble II (IUT Grenoble II). Elle est chercheure au CERAG UMR CNRS-UPMF. Ses travaux portent sur la veille stratégique et les stratégies de croissance des PME.
\end{abstract}

BÉRANGĖRE DESCHAMPS est maitre de conférences à l'IAE de Grenoble. Elle effectue sa recherche au CERAG. Ses travaux portent sur les profils de dirigeants, la croissance des PME innovantes et la reprise d'entreprise. Elle est l'auteure, avec Robert Paturel, de Reprendre une entreprise: de l'intention à l'intégration, Dunod, 2009.

SÉBASTIEN GEINDRE est maître de conférences à l'IAE de Grenoble où il enseigne le management et le management stratégique. II est chercheur au CERAG. Ses travaux portent sur les réseaux stratégiques, les stratégies de coopération locale des PME et le rôle du dirigeant dans la construction de la stratégie de l'entreprise.

\section{RÉSUMÉ}

À travers l'étude de quatre entreprises dans le secteur de la haute technologie, les auteurs s'intéressent au rôle joué par le dirigeant sur la stratégie de croissance. Une attention particulière est portée sur le profil du chercheur primo-entrepreneur.

Dans un premier temps, les critères de mesure de la croissance sont discutés et mis en relation avec le lien entre dirigeant et croissance; dans un deuxième temps, la méthodologie et les quatre cas étudiés sont présentés. Enfin, les résultats sont exposés en soulignant les liens entre profil de dirigeant et croissance. 


\begin{abstract}
Through the study of four hi-tech firms, the authors are interested in the role played by the leader on the strategy of growth. A particular attention is paid to the profile of the researcher firstly entrepreneur.

At first, we discuss growth's criteria of measure and we relate them with entrepreneur's profile and his attitude toward growth. Secondly, the methodology and four studied cases are presented. Finally, the results are exposed through the links between leader's profile and growth.
\end{abstract}

\title{
RESUMEN
}

A través del estudio de cuatro empresas en el sector de la alta tecnología, los autores se interesan en el papel jugado por el dirigente sobre la estrategia de crecimiento. Se centran más precisamente en el perfil del investigador (universitario) que llega a ser empresario.

Primero, tras un análisis crítico de los criterios de medida del crecimiento, dichos criterios son examinados a partir del vínculo entre dirigente y crecimiento; a continuación se presentan la metodología y los cuatro casos estudiados y por fin, se exponen los resultados a través de los vínculos entre perfil de dirigente y crecimiento.

\section{ZUSAMMENFASSUNG}

Die Einflussnahme des Geschäftsführers auf die Wachstumsstrategie wurde anhand von vier Fallstudien von Unternehmen im Hightech-Bereich untersucht. Besondere Aufmerksamkeit wurde dabei auf das Profil des Forschers und Jungunternehmers gelegt.

In einem ersten Schritt werden die Kriterien zur Messung des Wachstums diskutiert und anschliessend in Beziehung zum Geschäftsführer und zum Wachstum gesetzt. In einem zweiten Teil wird die Methodik erläutert und die vier Fälle beschrieben, schliesslich werden die Resultate dargelegt.

\section{Introduction}

La problématique de la croissance des entreprises, en particulier des PME, est un sujet de réflexion ancien, mais sans cesse réactivé soit par le monde académique (Sauvé, Majhoub et Pelletier, 2007; Julien, 2001), soit par le pouvoir politique (déclarations récentes en faveur du financement de la croissance de la $\mathrm{PME}^{1}$ ou les travaux du conseil d'analyse économique (voir notamment Betbeze et Saint-Étienne, 2006). La valorisation de la recherche publique par la création d'entreprise ou création d'activités innovantes est au cœur des nouveaux leviers de croissance coordonnés par l'État français et les collectivités territoriales : lois de 1999 et $2001^{2}$, mises en place des pôles

1. Rapport Novelli (Hurel, 2008) sur les croissances des PME (à travers les croissances externes dopées par les transmissions d'entreprise).

2. La loi no 99-587 sur l'innovation et la recherche du 12 juillet 1999 a pour objet de favoriser le transfert de technologies de la recherche publique vers le secteur économique et la création d'entreprises innovantes (cette loi a fait l'objet d'une 
de compétitivité, création des incubateurs publics, fusion des fonds dédiés par la Caisse des dépôts et consignations, concours de création d'entreprise innovante, mesures de défiscalisation pour les investissements en fonds propres dans les PME ou réorganisation d'OSÉO'.

Aujourd'hui, pour éviter le saupoudrage des moyens et les concentrer sur des cibles à fort potentiel de croissance, OSÉO est contraint à une plus grande sélectivité des projets, en particulier dans le domaine de l'innovation. Dans ce contexte, notre programme de recherche avec OSÉO et la région XY vise in fine à favoriser la mise en œuvre de nouveaux outils de sélection. L'hypothèse de notre recherche repose sur l'idée que le profil du dirigeant, de l'entrepreneur en particulier, notamment son seuil de compétences et sa capacité à ouvrir le capital de son entreprise, peut constituer un plafond de verre à la croissance par-delà les opportunités du marché. Le dirigeant façonne le modèle économique de son projet et, partant, la stratégie de croissance de l'entreprise. En d'autres mots, dans quelle mesure le profil de l'entrepreneur (ses caractéristiques, ses besoins) oriente-t-il le processus de croissance de son entreprise? Un dirigeant qui entreprend fait des choix stratégiques qui vont orienter et configurer son entreprise en lien avec l'ambition de son projet et eu égard à ses compétences et à son ouverture à un contrôle externe. Le postulat est que la croissance ne s'impose pas nécessairement à lui et reste son choix stratégique. Cette recherche de la croissance traduirait donc la mise en action de son intention stratégique (Hamel et Prahalad, 1989). Plus récemment, Teece (2007) propose d'une façon plus générale, c'est-àdire en intégrant la grande entreprise, un modèle fondé sur le management entrepreneurial de la firme en croissance rentable et durable. Les capacités dynamiques sont d'abord entrepreneuriales: détecter, favoriser et saisir les opportunités pour développer un modèle économique efficace basé sur des ressources et des actifs tangibles et intangibles. Les fonctions managériales d'analyse et d'optimisation deviennent des compétences de second rang.

Cette contribution est ici une première étape de notre programme de recherche. Dans le cadre d'une démarche abductive, l'objet est de dégager des idéaux-types de chercheurs primo-entrepreneurs dans des projets innovants,

codification aux articles L413-1 et suivants du Code de la recherche). La loi instaure un cadre juridique conciliant à la fois la participation des personnels de la recherche publique à la création et au développement d'entreprises, le fonctionnement régulier des services employant ces fonctionnaires et la moralité de leurs comportements. Les personnels concernés: les fonctionnaires civils des services publics et entreprises publiques dans lesquels est organisée la recherche, ainsi que certains personnels non fonctionnaires visés au décret $\mathrm{n}^{\circ}$ 95-168 du 17 février 1995 et no 2001-125 du 6 février 2001.

3. Établissement public français, OSÉO exerce trois métiers : l'aide à l'innovation, la garantie des concours bancaires et des investisseurs en fonds propres et le financement en partenariat. 
une des cibles d'OSÉO dans ses programmes de financements. Mustar (1998) avait souligné trois qualités du chercheur public pour conduire des projets entrepreneuriaux: capacité de conviction, ouverture sur l'international et aptitude au travail en équipe. Dans ce travail, l'idée est d'expliquer la relation entre le chercheur primo-entrepreneur et la croissance de l'entreprise. La recherche porte sur le processus de croissance de quatre entreprises innovantes qui ont en commun d'avoir été créées à la suite de projets de recherche développés au sein de leur laboratoire public, projets portés par les futurs fondateurs.

La première partie recouvre une présentation du concept de croissance et traite du lien entre le profil des dirigeants et le comportement organisationnel de leur entreprise, en mettant l'accent sur les stratégies de croissance. La finalité est d'établir un lien entre l'influence du profil du dirigeant sur la nature et les modalités de croissance de son entreprise. La deuxième partie s'articule autour de la présentation de notre méthodologie et des quatre études de cas. Enfin, la troisième partie vise à corroborer la pertinence de notre hypothèse théorique en établissant des liens entre le profil de ces dirigeants chercheurs primo-entrepreneurs et les choix de stratégie de croissance.

\section{Réflexion sur le rôle du dirigeant dans le processus de croissance}

La croissance des entreprises est un phénomène complexe. D'abord, elle est multidimensionnelle car elle est mesurable à partir d'indicateurs divers et non systématiquement corrélés (Davidsson et Wiklund, 1999; Delmar, Davidsson et Gartner, 2003; Vanacker et Manigart, 20064): augmentation du chiffre d'affaires sans forcément progression de l'emploi et inversement; croissance du chiffre d'affaires et dégradation de la valeur ajoutée. Ensuite, elle est multifactorielle dans le sens où il n'existe pas un facteur causal unique mais une conjonction de facteurs favorables: intention stratégique, ressources disponibles, attentes du marché, entre autres (Verstraete et Saporta, 2006). Enfin, le processus de croissance des entreprises n'est pas linéaire mais constitué de phases d'expansion, de consolidation, voire de recul ponctuel (le choix de la période de mesure est dans ce cas déterminant). Cette complexité pose donc naturellement la question du choix des indicateurs de mesure (1.1). Au-delà des critères de mesure, la croissance des entreprises se manifeste de manière différente selon les entreprises: elle peut être subie par le dirigeant (face aux contraintes du marché), voulue (par un dirigeant dont le projet

4. Ces auteurs ont opté pour une mesure multidimensionnelle de la croissance: chiffre d'affaires, nombre d'employés, total de l'actif, fonds de roulement, valeur ajoutée. Ces mesures sont à la fois prises de manière absolue et relative. 
initial portait l'ambition de la croissance) ou refusée (par un dirigeant qui ne souhaiterait pas, par exemple, ouvrir son capital à des investisseurs). Très liée au dirigeant, cette décision de croissance est étudiée sous l'angle de l'influence du dirigeant, en n'ignorant pas l'impact de l'environnement (1.2), ni les caractéristiques du profil du chercheur entrepreneur (1.3).

\subsection{Les indicateurs de la croissance et la problématique de leur mesure}

Une façon simple de présenter la croissance est de la ramener à une mesure. La croissance est donc une variation positive d'un indicateur donné. Les indicateurs élémentaires de mesure de la croissance en management stratégique sont souvent le chiffre d'affaires et l'emploi (Dunkelberg et Cooper, 1982; Almus, 2002). L'emploi est le plus pertinent du point de vue sociétal et les ventes, le plus pertinent au regard du dirigeant (Janssen, 2005); ces deux critères n'étant pas toujours liés. D'autres critères mesurent la croissance: soldes intermédiaires de gestion, valeur de l'actif net, fonds propres, brevets, parts de marché, profits, production physique de l'entreprise (Delmar, Davidsson et Gartner, 2003) ou encore fonds de roulement (Vanacker et Manigart, 2006). Ces indicateurs, parfois non corrélés, peuvent cependant se révéler insuffisants pour caractériser la croissance des entreprises, dans des contextes particuliers comme celui de l'innovation technologique, notamment des biotechnologies (Boissin et Trommetter, 2003). Ce point-là doit retenir notre attention compte tenu du caractère innovant des cas étudiés. Comme le note Julien (2001), la prise en compte du nombre de brevets déposés, des relations nouées par l'entremise du conseil scientifique, par exemple, sont autant de paramètres pris en compte notamment par les investisseurs pour apprécier le développement d'une organisation de ce type. Si l'absence de consensus (Weinzimmer, Nystrom et Freeman, 1998) sur le choix d'un critère semble évidente, nous retenons toutefois deux conclusions :

1. Le chiffre d'affaires, l'emploi et le volume d'actifs sont des indicateurs incontournables mais parfois insuffisants pour rendre compte de la croissance d'une firme;

2. Ils nécessitent de rester vigilant et ouvert à l'opportunité de «mesurer» d'autres critères dans le contexte d'entreprises impliquées dans le secteur des hautes technologies (brevet, relations, présence à l'international).

Au-delà du choix des indicateurs évoqués précédemment, la mesure de la croissance pose d'autres problèmes. En effet, celle-ci est très sensible à l'outil et à la formule de mesure (Delmar, Davidsson et Gartner, 2003). Il 
est donc nécessaire de proposer une réflexion sur 1) la nature de la mesure choisie (relative ou absolue),2) la période de mesure et 3 ) le choix éventuel d'un point de comparaison.

1. Comme le soulignent Vanacker et Manigart (2006), le fait de mesurer la croissance de manière absolue ou relative va biaiser les comparaisons si les tailles des entreprises mesurées sont très différentes: les mesures relatives vont avantager les petites structures (et donc les entreprises plus jeunes), les mesures absolues favoriseront les entreprises de grande taille. Une solution simple pour éviter une représentation faussée de l'importance de la croissance consiste à présenter les deux valeurs pour chaque entreprise et à travailler sur des entreprises de taille proche (vision privilégiée dans cette recherche).

2. Les modèles de métamorphose expliquent que la croissance n'est pas un phénomène linéaire (Greiner,1986; Churchill et Lewis, 1983). Entre des stades de croissance s'intercalent des moments de pause, voire de crises (Torrès, 1998). La croissance de l'organisation peut être vue comme une succession de phases de créativité et de consolidation. En conséquence, la croissance d'une entreprise, par exemple relativement à son secteur ou à l'économie, peut être faible ou nulle sur une courte période correspondant à une phase de consolidation, à un seuil, mais demeurer forte sur un intervalle plus long. À l'inverse, la croissance peut paraître artificiellement forte sur une courte période (notamment au moment d'une création) et finalement proche du rythme de l'économie ou du secteur sur un cycle plus long. Dans notre approche, la croissance est mesurée sur l'ensemble de la durée de vie des entreprises étudiées.

3. Le problème du point de comparaison est particulièrement saillant si les chercheurs souhaitent étudier des entreprises ayant des performances particulières (les entreprises à forte croissance, par exemple). Il est alors nécessaire de définir un référentiel légitime. Ne nous positionnant pas dans une logique d'étude d'entreprise à forte croissance mais cherchant à comprendre le processus générique des stratégies de croissance d'entreprises innovantes, ce point n'est pas développé dans cette recherche.

\subsection{Le rôle clé du dirigeant dans le comportement organisationnel de la firme}

Le dirigeant est un facteur explicatif clé du comportement de la firme. Ainsi, l'entrepreneur schumpétérien illustre ce point puisqu'il est présenté comme 
le vecteur clé de l'innovation et un facteur décisif dans le processus de croissance économique. Il en va de même pour les travaux qui posent le dirigeant en tant qu'acteur central du processus stratégique de l'entreprise (l'école entrepreneuriale décrite par Mintzberg, Ahlstrand et Lampel, 1999). Hamel et Prahalad (1989) ont développé le concept d'intention stratégique comme un état futur de l'organisation basé sur les aspirations du dirigeant et de ses membres afin de les motiver. Au-delà des opportunités de croissance et de l'organisation, la vision des dirigeants ou des futurs entrepreneurs façonne les orientations stratégiques (Varraut, 1999; Verstraete, 2001) donc nécessairement les chemins de croissance. Enfin, cette notion d'entrepreneur permet de réintroduire le créateur ou l'inventeur, figure manquante dans le modèle contractualiste selon Gomez (1996). La mise en acte des développements de la théorie de l'agence (Jensen et Meckling, 1976) dans les processus de contrôle des dirigeants par les actionnaires va dans ce sens: l'actionnaire corrige l'asymétrie informationnelle dont bénéficie le dirigeant auquel est délégué le pouvoir de gestion et qui bénéficie d'un pouvoir discrétionnaire pour maximiser sa seule utilité au détriment de l'actionnaire. C'est bien le dirigeant qui oriente la stratégie, qui propose une organisation adaptée et qui est donc l'acteur qui provoque, induit le comportement organisationnel.

La littérature propose de nombreuses réflexions sur la relation entre le profil du dirigeant et le comportement de la firme. Parmi elles, les travaux sur les typologies d'entrepreneurs ou dirigeants reposent sur l'hypothèse qu'à certains profils de dirigeants correspondent certains comportements de firme. Le travail de Daval, Deschamps et Geindre (2002) propose une synthèse de ces approches. Les auteurs ont déconstruit 25 typologies de dirigeant/entrepreneurs pour mettre au jour les caractéristiques sur lesquelles les auteurs des typologies fondaient leurs réflexions. La grille de lecture à laquelle les auteurs aboutissent décrit les liens entre l'individu et l'organisation (figure 1).

La conclusion des auteurs est la suivante: le comportement organisationnel (révélé par les décisions de politique générale et le management en place dans l'entreprise) est une réponse à une attente du dirigeant entrepreneur. Le développement (choisi donc) de la firme cherche à satisfaire un ou des besoins de son dirigeant. Certains profils d'acteurs développent une philosophie propre («orientation générale»: ce qui inclut les choix en matière de croissance) et un style de «management» en conséquence; l'environnement (marché, État, etc.) agissant comme une somme de facteurs de contingence favorisant ou inhibant le comportement attendu de la firme. Cette structuration concorde avec la typologie proposée par Storey en 1994 au sujet des déterminants les plus admis du développement des jeunes entreprises technologiques et classés en trois catégories:1) les caractéristiques propres à la firme;2) les caractéristiques des fondateurs;3) les caractéristiques externes (Bernasconi et Moreau, 2003). 


\section{FIGURE 1}

Modèle simplifié de la grille de lecture des typologies

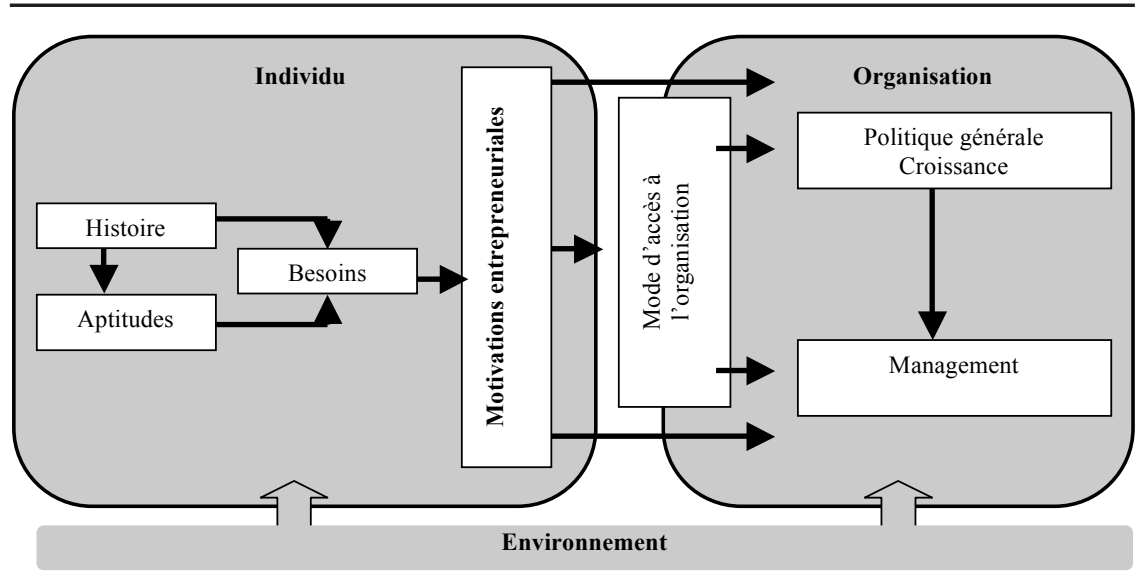

Source: Daval, Deschamps et Geindre, 2002.

La croissance qui apparaît dans le schéma de Daval, Deschamps et Geindre (2002) comme une composante du comportement organisationnel manifeste l'existence d'une relation spécifique entre profil de dirigeant entrepreneur et croissance de la firme. Plusieurs typologies mettent, en effet, en avant de manière spécifique cette relation (Laufer, 1975; Dunkelberg et Cooper, 1982; Miner, 1990; Julien et Marchesnay, 1987).

Comme le souligne Sammut (1996,p. 20), la croissance de la firme dépend de «la conscience stratégique du dirigeant et de l'ensemble des composantes internes et externes de l'organisation». Pour cette auteure, l'entrepreneur est au cœur de la transformation de l'entreprise qu'il dirige (p. 48). Ainsi, la croissance est le résultat d'une véritable décision de gestion dont l'entrepreneur propriétaire porte la responsabilité (Verstraete et Saporta, 2006). Le dirigeant impulse, en effet, les orientations stratégiques pour son entreprise (Verstraete, 1999). Selon Bernasconi et Moreau (2003), la stratégie existe tout d'abord dans l'esprit du leader comme une orientation à long terme, une vision de l'avenir et des résultats de l'organisation. Ces auteurs soulignent bien l'impulsion initiale du dirigeant sur le comportement de la firme. Ces mêmes auteurs affirment plus loin que les processus de développement qui seront mis en œuvre par les dirigeants permettront d'atteindre ou non le développement souhaité. C'est donc le dirigeant qui initie la croissance et met en œuvre des actions pour l'atteindre, en particulier dans le contexte d'une jeune entreprise, dont il est à l'origine de la création. Ces auteurs élaborent un modèle permettant de classer les entreprises technologiques en trois catégories selon la nature du contrôle souhaité par les entrepreneurs et la dynamique potentielle du projet. 
Est introduite ici une première composante du profil du dirigeant: selon son acceptation du contrôle par un tiers, la trajectoire de l'entreprise (sa croissance en l'occurrence) sera différente. Ces auteurs évoquent également le niveau d'ambition du projet (p. 19), autre élément du profil de l'entrepreneur.

Le profil du dirigeant (ou de l'entrepreneur) est caractérisé dans la documentation sur les typologies par quatre composantes:1) l'histoire de l'entrepreneur, 2) ses aptitudes et 3) ses besoins concernant l'entrepreneur en tant qu'individu. Celui-ci est guidé dans son action entrepreneuriale par 4) des motivations plus ou moins prégnantes qui résultent de la combinaison des variables précédentes. Des liens existent entre ces quatre composantes (Daval, Deschamps et Geindre, 2002). L'histoire de l'entrepreneur rassemble les caractéristiques classiques de l'individu telles que l'âge, le sexe, la formation, l'expérience professionnelle, l'éducation, le tissu relationnel, la détention de capital et l'entourage entrepreneurial. Cette catégorie conditionne les aptitudes entrepreneuriales de l'individu qui renvoient essentiellement aux traits de caractère de l'entrepreneur (confiance en soi, esprit de compétition, esprit d'initiative, ambition, attitude à l'égard du risque, etc.). Les aptitudes conditionnent, en complément de son histoire, la nature des besoins de l'individu (sécurité, reconnaissance, pouvoir, contrôle, réalisation de soi), considérés par les auteurs comme l'une des conditions sine qua non de l'acte d'entreprendre. Les motivations, capitales pour la conduite de l'opération, influencent les décisions que les entrepreneurs adoptent pour démarrer leur entreprise et également le comportement ultérieur des entrepreneurs dans la définition de leur politique de croissance: «la motivation est identifiable dès le stade du projet mais conditionne aussi l'évolution ultérieure de l'entreprise» (Laufer, 1975, p. 13).

Une interaction entre la personnalité de l'entrepreneur et les conditions de développement de son entreprise est démontrée. Il existe donc une relation directe entre ce qui constitue le profil de l'individu (son histoire, ses aptitudes, ses besoins et ses motivations) et la façon dont il va conduire la firme. Lorrain et Dussault (1988) mettent d'ailleurs en évidence les liens entre profil de l'individu (besoins et histoire) et comportements de gestion (désir de croissance, professionnalisme de la gestion et de la structure). Dans la continuité, des études qualimétriques montrent l'importance de l'influence du dirigeant, qui, poussé par sa volonté de croître, obtient des combinaisons d'avantages concurrentiels, développe des produits, dépose des brevets et met en place des dispositifs de veille afin de transformer les menaces en opportunités (Audet, 2003; Chalus-Sauvannet, 2006).

Parmi les réflexions existantes sur les comportements organisationnels de l'entreprise, nous privilégions pour l'heure l'influence du profil du dirigeant sur la stratégie de croissance de l'entreprise en omettant l'influence 
de l'environnement, des parties prenantes et des ressources et compétences présentes dans ces quatre dimensions (figure 2). De la même façon, les relations d'influence sont réflexives entre acteurs, organisations, parties prenantes et environnement. Dans cette contribution, l'objet d'étude est circonscrit à un certain déterminisme (flèche en gras dans un seul sens) de la vision du dirigeant sur la stratégie de croissance, c'est-à-dire à une vision réductionniste face à la complexité du processus de construction de la stratégie de croissance.

FIGURE 2

\section{L'objet étudié}

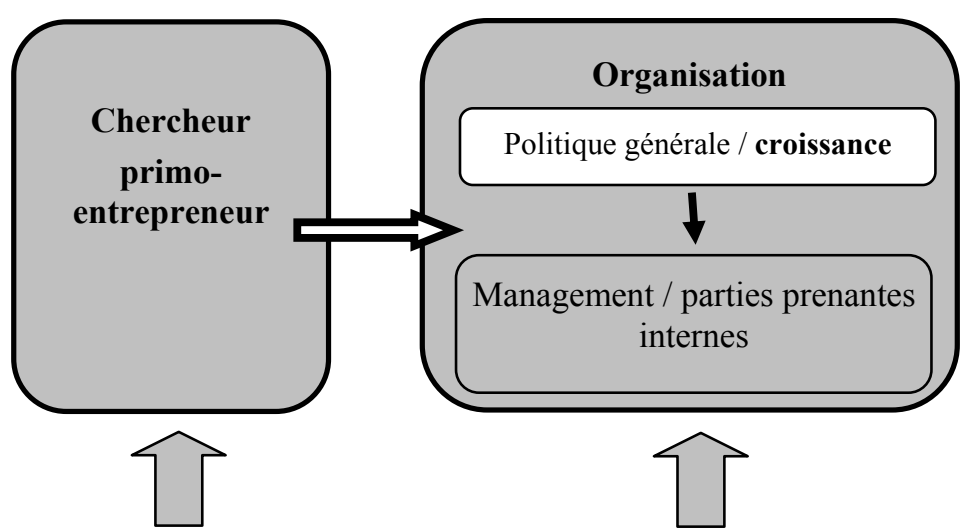

\section{Environnement / parties prenantes externes}

\subsection{Le profil et la naissance du chercheur entrepreneur}

La loi du 12 juillet 1999 en France encourage et encadre la création d'entreprise par les chercheurs publics. En effet, la valorisation de la recherche en est l'objectif. Même si Émin $(2003,2006)$ s'est interrogée sur l'intention d'entreprendre, Philippart (2008) relève le faible nombre de recherches portant sur la création d'entreprise par des chercheurs fonctionnaires. Il suggère des pistes de recherche intéressantes sur cette thématique. Notre présent article traite de la place du chercheur dans la firme qu'il a lancée (Philippart, 2008, p. 14). Avant la loi, quelques références traitaient du chercheur entrepreneur. Celui-ci, également appelé «entrepreneur académique» (Doutriaux, 1994), s'oriente vers la création d'entreprise de haute technologie, d'informatique, d'électronique ou encore de matériaux spécifiques. Ces secteurs, d'après Bertholon (1994), disposent de caractéristiques communes (adaptées aux possibilités d'investissements en compétences et en finance des universi- 
taires): 1) des secteurs qui n'ont pas besoin d'investissement en production trop important;2) des secteurs à haute valeur ajoutée technologique et dont l'investissement immatériel (la compétence) est très important;3) des secteurs spécifiques sur lesquels le marché est limité en volume, même s'il est étendu en termes géographiques.

Il observe aussi que les entreprises qui possèdent le plus fort taux de croissance potentiel proviennent de grandes universités, actives dans les partenariats industriels. Notons que ces chercheurs entrepreneurs ont souvent un profil d'ingénieur diplômé. Ces ingénieurs entrepreneurs sont porteurs d'innovations et générateurs de richesse (Fayolle, 1994). Ils sont issus d'écoles différentes dans un système français très hiérarchisé avec des notoriétés très variables selon les établissements. Ainsi, le niveau de l'école mais aussi son programme d'enseignement (management, finance, entrepreneuriat, etc.) peuvent avoir un impact sur le profil du futur chercheur entrepreneur.

Le chercheur est aujourd'hui incité à valoriser ses recherches par la création d'entreprise. Pour autant, quelle est la place de ce chercheur primoentrepreneur dans le développement de l'entreprise qu'il a créée? D'un point de vue théorique, la croissance d'une entreprise dépend, toutes choses égales d'ailleurs, du profil de dirigeant, objet de l'analyse qui va suivre sur quatre études de cas basées sur des chercheurs ayant fondé leur entreprise.

\section{Une méthodologie de recherche abductive}

Si la croissance est bien le résultat d'une combinaison de facteurs favorables (les attentes du dirigeant associées à divers facteurs de contingence environnementaux ou organisationnels), le rôle spécifique du dirigeant mérite d'être exploré. Il convient toutefois d'être prudent et de conserver à l'esprit la dimension multifactorielle de la croissance. En conséquence, il s'agit de proposer un protocole de recherche permettant à la fois d'étudier la relation dirigeant-croissance tout en essayant d'assurer un contrôle raisonnable des autres variables (environnement, organisation, parties prenantes). Notre analyse suit un mode de raisonnement abductif qui vise à produire ou renforcer notre hypothèse centrale. Nos données, essentiellement qualitatives et structurées par des études de cas, sont particulièrement adaptées à notre objectif de recherche qui vise à mieux comprendre la nature et les modalités de croissance des entreprises fondées par des chercheurs primo-entrepreneurs.

\subsection{Le choix de l'abduction et de l'étude de cas}

L'abduction consiste à élaborer une observation empirique qui relie une règle générale à une conséquence. Elle permet de retrouver la conséquence si la règle générale est vraie (David, 1999). «L'abduction est l'opération qui, 
n'appartenant pas à la logique, permet d'échapper à la perception chaotique que l'on a du monde réel par un essai de conjecture sur les relations qu'entretiennent effectivement les choses» (Blaug, cité par Koenig, 1993, p. 7). Ce mode d'inférence «consiste à tirer de l'observation des conjectures qu'il convient ensuite de tester et de discuter» (Koenig, 1993, p. 7). Pour Carontini (1990), la force et la faiblesse de ce raisonnement se situent précisément au niveau de la relation qui doit être établie entre ces deux prémisses que sont le résultat (une donnée d'observation) - ici les processus de croissance des entreprises observées - et la règle, le principe, la condition générale ou la cause, pouvant fournir une explication de ce résultat, et ce faisant, le transformer en un cas d'application de la règle générale - ici les connaissances théoriques sur les profils de dirigeant et la croissance. «La relation entre le résultat et la règle demeure donc une relation nécessairement hypothétique et conjecturale» (Carontini, 1990, p. 221). Cette approche nous apparaît pertinente pour deux raisons. En premier lieu, nous ne nous situons pas dans un contexte vierge en termes de connaissances. Une approche strictement inductive ne serait par conséquent pas appropriée. En second lieu, nous sommes dans un cadre exploratoire et avons pour objectif de renforcer notre hypothèse centrale (le profil du dirigeant influence la croissance de l'entreprise) tout en faisant émerger des hypothèses corollaires sur les liens spécifiques qui pourraient être mis au jour entre certains profils et les modalités de croissance qui leur sont associées.

Nous avons retenu une recherche d'informations réalisée au moyen d'entretiens semi-directifs avec guide 5 auprès des chercheurs fondateurs, d'observations directes (p. ex., visites de l'entreprise) et de collecte de données secondaires (bilans, plaquettes, rapports d'activité). La collecte des données s'est échelonnée sur six mois. Ces cas ont été retenus pour l'intérêt qu'ils présentaient a priori, pour leur exemplarité (Yin,1989). Les dirigeants interrogés ont été sélectionnés à partir de plusieurs critères: primo-chercheur entrepreneur, entreprise de haute technologie et innovante, homologie des parcours des créateurs (doctorat), croissance de leur entreprise sur au moins deux des critères classiques de croissance (chiffre d'affaires, fonds propres, effectifs et campagne de collecte de fonds). Notre objectif était de maîtriser autant que possible un certain nombre de critères de contingence (secteur d'activité, localisation géographique, parcours des dirigeants, etc.).

5. Le guide d'entretien de type semi-directif, et sous forme de questions ouvertes comportait les thèmes suivants: éléments d'information et compréhension de l'entreprise (thème 1 ), antécédents du dirigeant (thème 2 ), croissance de l'organisation (thème 3 ), vision stratégique du dirigeant pour l'entreprise (thème 4), gouvernance (thème 5) et stratégie d'innovation (thème 6). 


\subsection{Description des cas: entreprises et dirigeants}

Notre recherche s'appuie sur quatre cas d'entreprises. Ces PME, âgées de moins de 10 ans (créées entre 1997 et 2003), présentent des effectifs variant aujourd'hui entre 30 et 120 personnes (chiffres 2007) et un chiffre d'affaires se situant entre 0 et 15 millions d'euros. Les entreprises sont localisées dans une même zone géographique et ont bénéficié de la même structure de mise en relation entre porteurs de projet et financeurs. Enfin, les quatre entreprises commercialisent des produits destinés à entrer dans les processus de production de produits de grande consommation à l'échelle mondiale (aérospatiale, électronique grand public et électronique de pointe, cosmétique).

Ces quatre entreprises partagent a priori les points communs suivants: elles sont innovantes, très ouvertes à l'international et en croissance, cette croissance nécessitant une ouverture du capital.

Par leur caractère fortement innovant, nos cas d'entreprise développent principalement des innovations de type «destruction créative» (Schumpeter, 1939), voire des competence destroying: celles qui détruisent car elles reposent sur des savoir-faire totalement nouveaux selon Tushman et Anderson (1986). Ils conduisent des innovations de rupture en appliquant des technologies nouvelles susceptibles d'introduire des facteurs de succès pour leurs clients: création de nouveaux produits, de fonctionnalités différentes sur des produits existants, innovation majeure dans les process de production, etc. La forte croissance de leur secteur se double logiquement d'un risque important, celui notamment d'être concurrencé par une technologie de substitution.

Ces entreprises se positionnent toutes en business to business et leurs clients actuels ou potentiels sont mondiaux. Au-delà, certaines de ces entreprises disposent de structures organisationnelles en dehors de la France (filiales en Europe et aux États-Unis pour E1, filiale en Asie pour E3 et plateforme de production localisée aux États-Unis pour E4), voire d'un actionnariat et d'une équipe managériale internationale (cas de E1 et E4). «Je voulais conquérir le monde par ma clientèle, par mes actionnaires et aussi en implantant des filiales partout dans le monde» (dirigeant de E1).

Le tableau 2 synthétise les caractéristiques générales des dirigeants des quatre entreprises étudiées. Il met en lumière à la fois les points communs et les différences de ces entrepreneurs.

Les dirigeants des entreprises étudiées sont tous issus de la recherche publique. De profil ingénieur docteur, ils maîtrisent tous la technologie à partir de laquelle ils ont fondé leur entreprise. Ces descriptions des cas d'entreprises et d'entrepreneurs laissent apparaître des trajectoires différentes, en grande partie dues à des profils de dirigeants différents, dont nous discutons dans la partie suivante. 


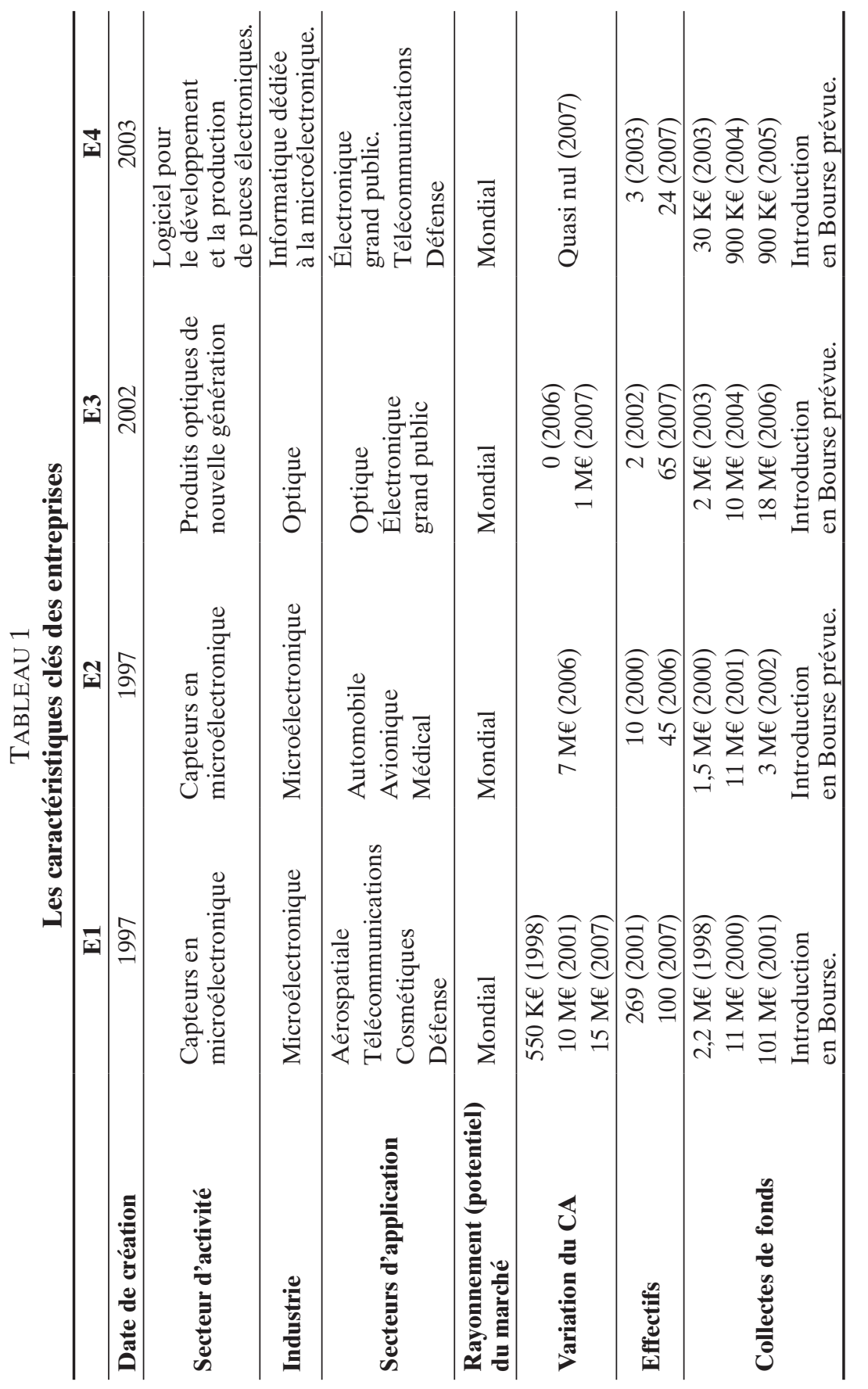




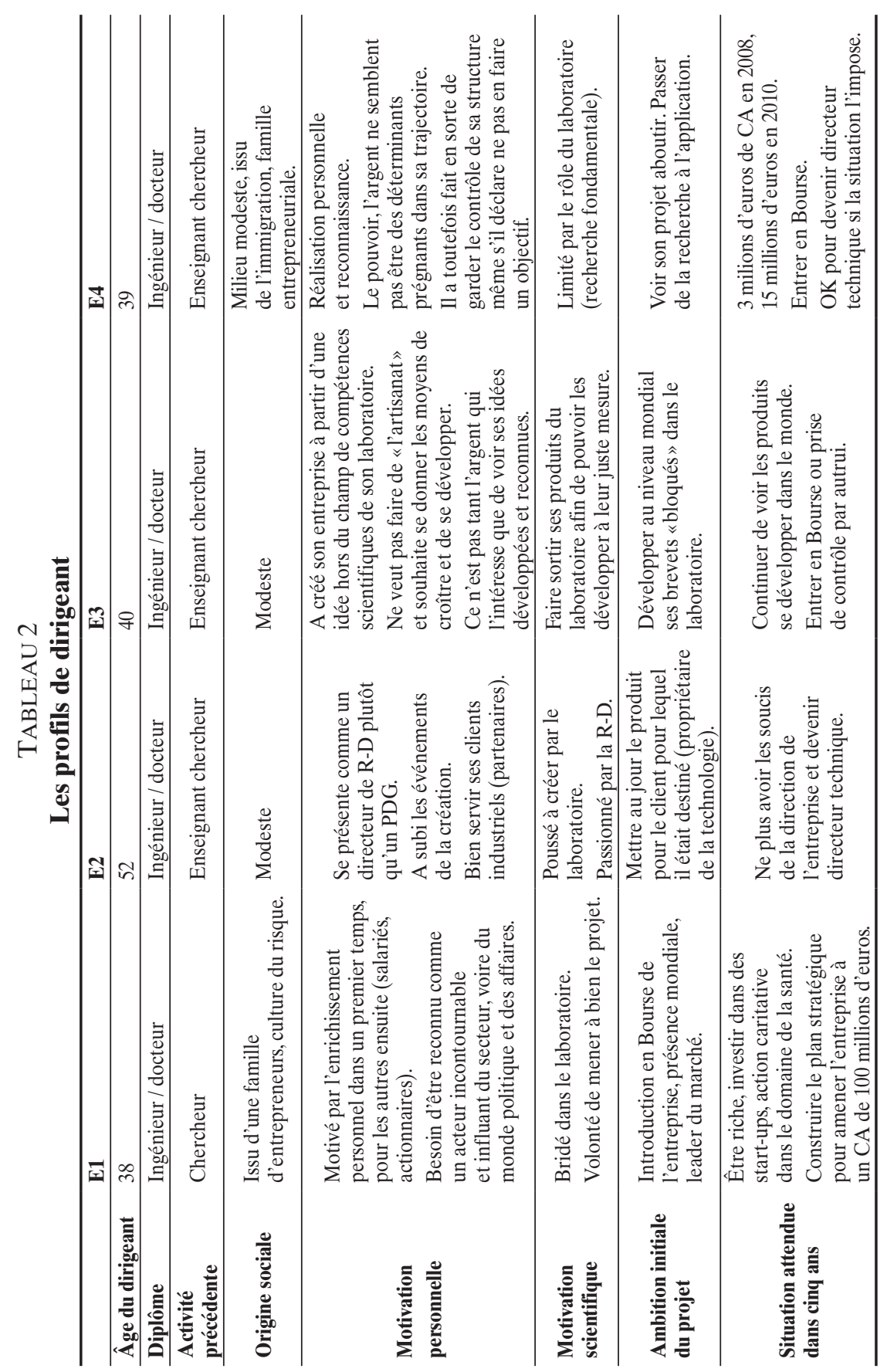




\section{L'influence du profil du dirigeant sur la trajectoire de l'entreprise}

En décrivant les cas, nous avons insisté sur les points qui rassemblaient ces dirigeants et ces entreprises. Toutefois, l'accent mis sur les différences tend à montrer combien le profil du dirigeant influence la nature de la croissance de l'entreprise. Le cas E1, en particulier, présente une évolution bien différente de celle des trois autres.

\subsection{Convergences et spécificités des trajectoires de croissance}

Les quatre entreprises de haute technologie étudiées ont suivi des trajectoires différentes. E1 a progressé très rapidement sur l'ensemble des critères de mesure (effectif, CA, fonds propres, internationalisation, brevets), plus rapidement que E2, pourtant sur le même segment d'activité, mais dont la croissance est plus stable et moins intense. E1, en effet, a dû subir une restructuration à la suite de l'effondrement de son marché principal et a su redresser la situation en une année. E1 et E2 présentent des résultats positifs après 10 ans d'activité. Selon nous, la différence de profil du dirigeant, et notamment ses ambitions, peut expliquer une partie des spécificités de ces deux trajectoires de croissance. L'un (E2) a été poussé à créer pour ne pas perdre la technologie, l'autre (E1) est davantage proactif dans sa décision de créer et dans le projet qu'il a porté. E3 et E4 connaissent des modes de croissance assez proches en termes de régularité et en matière de structuration de l'entreprise.

Nous constatons que l'expression de la croissance ne se traduit toutefois pas forcément sur les trois critères classiques (chiffre d'affaires, fonds propres, effectifs), la croissance du CA pouvant être en décalage temporel avec la croissance des deux autres critères (cas E3 et E4, ou E1 et E2 dans leurs premières années d'existence). La croissance des fonds propres constitue le moyen de faire croître l'effectif, afin de s'assurer une espérance de gain liée à la réalisation future du CA. Le rôle du dirigeant dans la croissance consiste, par conséquent, à fournir des perspectives futures de performance de l'entreprise attendue par les investisseurs. La nature de ces garanties (dépendantes du niveau de risque et donc de la quantité de ressources mobilisées par les investisseurs) évolue dans le temps. Or, la capacité à fournir ces garanties va dépendre directement du dirigeant, de ses qualités propres, de son expérience, de son entourage, mais aussi de sa volonté à s'investir dans cette tâche de persuasion.

En effet, la densification des réseaux formels traduit le développement de l'entreprise (apparition d'administrateurs nouveaux, structuration du conseil scientifique). Ces éléments, au même titre que l'augmentation du chiffre d'affaires, sont des indicateurs traduisant un développement «harmonieux», dans lequel les investisseurs peuvent avoir confiance, s'impliquer 
et, par là même, renforcer le processus de croissance (nouvelles collectes de fonds qui permettent le renforcement de l'équipe). E1, par exemple, consolide son conseil d'administration en fonction de ses innovations en recherchant des experts et donc un réseau susceptible de développer le marché de ce nouveau produit. Ainsi, E3 nous explique: «mon projet est ambitieux, je ne veux pas faire de l'artisanat; j'ai choisi un investisseur qui avait le même esprit que moi, cela n'aurait pas pu être autrement».

\subsection{L'influence des profils de dirigeant sur les stratégies de croissance}

Le tableau suivant permet de synthétiser les attentes et les enjeux des dirigeants et de mettre en parallèle le type de croissance de ces entreprises ainsi que leurs relations avec les parties prenantes. Certaines convergences peuvent alors être observées.

TABLEAU 3

Relation profil du dirigeant et stratégie de croissance

\begin{tabular}{|c|c|c|}
\hline \multicolumn{3}{|c|}{ Attentes du dirigeant } \\
\hline & E1 & E2, E3 et E4 \\
\hline Enjeu de l'entreprise & Enjeu commercial & Enjeu technologique \\
\hline Enjeu personnel & $\begin{array}{l}\text { Entreprendre pour le profit, } \\
\text { la réussite individuelle. }\end{array}$ & $\begin{array}{l}\text { Entreprendre pour } \\
\text { faire aboutir une idée, } \\
\text { un projet, un produit. }\end{array}$ \\
\hline $\begin{array}{l}\text { Entreprise lieu } \\
\text { de création de }\end{array}$ & Richesse & Savoir \\
\hline \multicolumn{3}{|c|}{ Impact sur la croissance } \\
\hline $\begin{array}{l}\text { Intensité } \\
\text { de croissance }\end{array}$ & $\begin{array}{l}\text { Forte amplitude. Risque de } \\
\text { perte de contrôle du processus } \\
\text { de croissance (restructuration). }\end{array}$ & $\begin{array}{l}\text { Amplitude modérée. } \\
\text { Maîtrise du processus } \\
\text { de croissance. }\end{array}$ \\
\hline Modalités & $\begin{array}{l}\text { Croissance interne } \\
\text { et croissance externe. }\end{array}$ & $\begin{array}{l}\text { Croissance interne } \\
\text { et croissance conjointe } \\
\text { (coopération). }\end{array}$ \\
\hline Prise de risque & $\begin{array}{l}\text { Sur le marché et sur le } \\
\text { développement des produits. }\end{array}$ & $\begin{array}{l}\text { Sur le développement } \\
\text { du produit. }\end{array}$ \\
\hline \multicolumn{3}{|c|}{ Relation avec les parties prenantes } \\
\hline Marché & Concurrents, clients & Réseau de partenaires \\
\hline Actionnaires & $\begin{array}{l}\text { Investisseurs } \\
\text { Attendent un retour } \\
\text { sur investissement élevé } \\
\text { (y compris le dirigeant). }\end{array}$ & $\begin{array}{l}\text { Partenaires } \\
\text { Participent à } \\
\text { l'élaboration du projet } \\
\text { scientifique. }\end{array}$ \\
\hline $\begin{array}{l}\text { Organe clé } \\
\text { de l'entreprise }\end{array}$ & $\mathrm{CA}=$ rôle prédominant. & $\begin{array}{l}\text { Comité scientifique = } \\
\text { rôle prédominant. }\end{array}$ \\
\hline
\end{tabular}




\section{Croissance et logique de création des entrepreneurs}

La première différence relevée tient à la place que prend l'entreprise dans le projet de vie de son dirigeant. Sur ce point, E1 fait montre d'une logique différente des trois autres dirigeants: créer son entreprise est un moyen de faire fortune afin de satisfaire des ambitions personnelles en dehors du champ d'application de son travail de recherche. E1 a davantage un profil de gestionnaire que les autres (à la connotation technique beaucoup plus exclusive). La création n'a pas été subie: c'est un vrai choix dont il a été le moteur. Il s'agit d'un véritable entrepreneur (au sens de Doutriaux, 19946). L'ambition du projet était importante dès le départ (l'introduction en Bourse était souhaitée et planifiée, de même que l'atteinte de 100 millions d'euros de CA). L'entreprise n'est qu'une étape dans sa carrière professionnelle lui procurant les moyens de réaliser ses ambitions personnelles; c'est un morceau d'un projet personnel plus vaste. Pour E2, E3 et E4, l'entreprise est au contraire une finalité pour leur projet (l'industrialisation d'une technologie expérimentale et la réussite d'un projet - scientifique - personnel). Les profils se ressemblent: des dirigeants, avant tout, scientifiques dans l'âme, motivés par l'application et la mise en pratique de travaux plus expérimentaux. L'entreprise est apparue comme un lieu plus propice au développement de leur projet que leur laboratoire, c'est un moyen, mais ce n'est pas la «chose » du dirigeant (il a été poussé à créer dans le cas de E2, aidé dans le cas de E4; E3 a créé en raison des «insuffisances» de son laboratoire, sinon, il y serait resté). Pour E2, le moins proactif des dirigeants dans la démarche entrepreneuriale, cela s'apparente même à un «mal nécessaire », car sans lui la technologie n'aurait pas été exploitée. L'entreprise est certes une finalité pour le projet mais pas pour son pilote dont l'aspiration managériale ne semble pas se révéler, même après plusieurs années de direction. Au contraire, il souhaite devenir directeur technique et scientifique. Dans les cas de E3 et E4, la fonction managériale qui en soi n'était pas un facteur motivant à la création (diriger, faire du profit) n'est pas non plus rédhibitoire. E4, par exemple, a toujours le contrôle du capital de sa structure. Il envisage sereinement qu'un jour il puisse devenir responsable technique et être amené à quitter la direction générale tout en étant satisfait de sa position actuelle. Dans le cas de E3, la forte croissance du capital l'a déjà conduit à céder sa place de dirigeant pour devenir directeur scientifique et se concentrer sur les activités de R-D. Cette perspective est vécue sereinement, dans «l'ordre des choses», pour permettre au projet d'atteindre l'ampleur que le dirigeant a envisagée. Dans tous les cas, la croissance est un moyen de satisfaire une attente personnelle

6. Chercheurs avec une expérience industrielle, proactifs, qui ne voulaient pas faire carrière à l'université ; leurs entreprises présentent le plus haut taux de croissance au lancement. 
du dirigeant (la réussite financière et sociale ou la réussite technologique). Toutefois, la nature de cette attente semble conditionner une croissance plus ou moins ambitieuse. Ainsi, nous pouvons formuler l'hypothèse corollaire que des dirigeants dont l'entreprise n'est qu'un composant d'un projet plus vaste (mais orienté vers une réussite personnelle) sont susceptibles d'induire des perspectives de croissance plus intenses.

\section{Croissance et rapports des entrepreneurs avec leur environnement}

La seconde grande différence que nous relevons dans les cas réside dans le rapport que les dirigeants ont instauré dans leur relation au marché (clients) et, d'une manière plus générale, aux différentes autres parties prenantes (actionnaires, concurrents, communauté scientifique). E1, à l'instar des trois autres dirigeants par ses produits et leurs usages, se place volontairement sur un secteur innovant, avec des perspectives de croissance mondiale importantes. Son ambition a toujours été de devenir «leader mondial» et la perspective commerciale a toujours accompagné, si ce n'est précédé, la perspective technologique. Dès la création, l'orientation de l'entreprise vers une croissance forte était planifiée. Le créateur a alors cherché à atteindre son objectif par une croissance aussi bien interne qu'externe de dimension internationale (11 filiales dans 11 pays différents). En outre, il a rapidement cherché à élargir les champs d'application de sa technologie afin de capter au plus vite la valeur, qu'un grand nombre de clients pouvaient apporter à son entreprise en termes de profit. L'entreprise a connu une croissance rapide, forte mais a dû subir des turbulences importantes (croissance très forte, restructuration, cession d'actifs, recherche de nouveaux secteurs d'application, recroissance). Les clients sont avant tout perçus comme des apporteurs de ressources financières et des sources de profit. Si E1 parle de clients, nos trois autres dirigeants évoquent, eux, des partenaires. Pour E2, la croissance commerciale est ici tirée par le marché. Elle est voulue, souhaitée et sécurisée par les partenaires commerciaux. Rappelons que le dirigeant a été poussé par son laboratoire d'origine et par les clients (auxquels était destinée la technologie) à créer son entreprise: il l'a subie. La trajectoire de cette entreprise, placée sur le même secteur que E1 a été plus harmonieuse, plus linéaire et n'a pas eu à essuyer de crise majeure. De même, la croissance de E3 est voulue et activée par la sollicitation directe de grands groupes internationaux très intéressés par les produits qu'elle développe. Grâce à ses partenariats, elle a ouvert une filiale et développé de la sous-traitance en Asie. Le fondateur est soutenu et accompagné par les partenaires régionaux. Le dirigeant et son équipe donnent une impression de très grande sérénité quant à leur développement et à leur avenir dans un contexte de perte de contrôle du capital et du poste de dirigeant par le fondateur: «je fais en sorte que mon 
entreprise soit prête pour une entrée en Bourse ou pour un rachat». Le cas de E4 va dans le même sens. Très tôt dans le développement de l'entreprise, celle-ci a installé un bureau aux États-Unis afin d'être à proximité de ses clients futurs, parties prenantes au développement du logiciel mais aussi des concurrents avec lesquels l'entreprise collabore. Dans les cas de E2, E3 et $\mathrm{E} 4$, la croissance se fonde donc largement sur des relations principalement coopératives (participation à différents projets, coopérations, y compris avec des concurrents) dans un souci de réussite du projet initial. Le risque paraît mesuré et la prise de risque, limitée (les partenariats avec les clients potentiels réduisent le risque commercial et les coopérations avec les concurrents réduisent le risque sectoriel). Ainsi, nous pouvons formuler une deuxième hypothèse corollaire: la recherche d'une croissance soutenue en lien avec un entrepreneur orienté profit semble favoriser la croissance externe en complément de la croissance organique. À l'inverse, les profils plus orientés "projet scientifique» semblent privilégier la coopération et la croissance conjointe en complément de leur croissance organique.

\section{Croissance et nature des liens avec le laboratoire}

Nous nous intéressons aux chercheurs primo-entrepreneurs. Ces chercheurs fonctionnaires ont quitté leur laboratoire pour créer une entreprise. Qu'en est-il a posteriori des liens qui les unissaient? E1 est en conflit avec son laboratoire d'origine, mais son ancien directeur de laboratoire est actionnaire de son entreprise; des liens amicaux les unissent. Le dirigeant E1 est propriétaire de son invention et a déposé de nombreux brevets; il n'a plus à collaborer avec ses anciens partenaires. E2 reste très attentif aux remarques de ses anciens collègues, par ailleurs très parties prenantes dans le conseil scientifique de l'entreprise et très impliqués dans les relations à la clientèle. Le fondateur d'E3 a un discours très positif sur sa relation et le rôle de ses tutelles scientifiques. Rappelons que son projet est issu d'une recherche hors des programmes de son laboratoire, ce qui peut ne pas être neutre. Il a développé par la suite des relations avec d'autres laboratoires liés à son projet hors du champ de son laboratoire originel. Il insiste sur le caractère confortable de sa démarche: il est en disponibilité et peut revenir à l'université même si ce n'est pas à l'ordre du jour. Pour E4, le laboratoire d'origine est actionnaire de E4 et codétenteur du brevet. Cette structure a joué et joue encore un rôle clé dans l'entreprise. Nous observons en troisième hypothèse corollaire que les chercheurs primo-entrepreneurs qui se sont le plus émancipés de leur laboratoire sont aussi ceux qui connaissent la croissance la plus forte (E1 et E3).

Il est intéressant de constater que les laboratoires d'origine ont facilité l'émancipation des chercheurs entrepreneurs. Toutefois, ils ne sont plus 
des parties prenantes clés dans le développement actuel des entreprises. Contrairement aux travaux de Gulati, Nohria et Zaheer (2000) qui montrent que les réseaux peuvent parfois inhiber le développement des organisations, les laboratoires - dans nos cas - n'ont freiné ni la croissance ni le développement de nouveaux réseaux (administrateurs, conseil scientifique, client, etc.).

\section{Conclusion}

L'objectif de cet article est d'étudier l'impact des profils de dirigeants sur la nature de la croissance de leur firme. Après avoir établi théoriquement le rôle clé du dirigeant dans l'orientation choisie en matière de croissance pour son entreprise, nous avons, à travers quatre études de cas, essayé de comprendre et d'expliquer les différentes trajectoires suivies par ces entreprises.

Afin d'isoler autant que possible la variable «profil de dirigeant» dans notre analyse, nous avons fait le choix de travailler à partir de quatre cas ayant en commun une activité innovante, hautement technologique, sur des secteurs fortement porteurs, internationalisés. Ces entreprises ont toutes été créées dans le même environnement régional, que nous pouvons qualifier de propice (présence d'un pôle de compétitivité référent, accès local à des capitaux-risqueurs d'envergure, bassin d'emploi hautement qualifié). En outre, nous avons choisi des entrepreneurs ayant comme point commun d'être des chercheurs (homogénéité des cursus) issus de laboratoires publics et créant une entreprise pour la première fois (homogénéité des expériences).

Alors que les environnements de ces entreprises se ressemblent, que les parcours de dirigeants sont proches et qu'a priori toutes ses entreprises sont orientées «naturellement» vers la croissance du fait même de leurs spécificités technologiques, il existe des formes de croissance différenciées. Notre analyse autorise à penser que le postulat formulé dans la première partie s'en trouve renforcé. En effet, nous constatons que les attentes du dirigeant vis-à-vis de son entreprise et du projet qu'elle représente semble agir à la fois sur l'intensité de la croissance et sur les modalités de celle-ci. Au-delà du profil entrepreneurial du dirigeant, le profil entrepreneurial du management, quelle que soit la taille de l'entreprise, semble devenir un élément prépondérant. Teece (2007) fait référence à un nouvel hybride, un capitalisme managérial entrepreneurial en élargissant au management de la grande entreprise les caractéristiques individuelles de l'entrepreneur lançant une nouvelle activité innovante.

Notre hypothèse centrale sur le lien entre profil de dirigeant et stratégie de croissance s'en trouve renforcée conformément à la logique abductive. Nos observations font ainsi apparaître deux types de profils de dirigeant: 
d'un côté, un individu dont l'entreprise est avant tout le lieu de la réalisation d'un projet personnel tourné vers la réussite individuelle, l'argent, la reconnaissance sociale et dont la croissance de l'entreprise est forte, voire excessive, parfois dangereuse pour la structure; de l'autre côté, un individu dont l'ambition est avant tout scientifique et l'entreprise, un moyen d'assurer la finalisation d'un projet de recherche, ce qui suppose une croissance plus modeste (mais réelle et nécessaire). Il est entendu que cette opposition peut paraître caricaturale mais reste conforme à de nombreux autres travaux (Doutriaux, 1994; Dunkelberg et Cooper, 1982; Miner, 1990; Marchesnay, 1998). Le lien théorie-terrain de l'abduction est ici vérifié. Là où nos résultats permettent d'aller plus loin sur deux points:1) en présentant ces deux cas non pas comme une opposition mais comme les deux bornes d'un continuum dont E1 et E2 seraient les représentants les plus caractéristiques (E3 et E4 apportant des nuances au modèle E2);2) dans la mise au jour de relation entre ces profils de dirigeants et les outils mobilisés pour assurer leur croissance (approche du marché, rôle attendu des actionnaires, mode de développement, organes clés de gouvernance). Ces travaux permettent de poursuivre notre protocole de recherche visant à tester les liens que nous avons établis entre profil de chercheurs primo-entrepreneurs et stratégies de croissance de la jeune entreprise innovante.

Enfin, dans le cadre d'une meilleure allocation des fonds publics en faveur des entreprises en croissance (OSÉO et Régions), il ressort que le profil du dirigeant peut être prépondérant dans le choix de la stratégie de croissance, indépendamment de l'environnement. Ainsi, le chercheur primoentrepreneur semble avoir un profil plus orienté vers la croissance que vers la pérennité (voir les profils PIC / CAP de Julien et Marchesnay, 1996). Il est vrai que le chercheur public possède, par son statut, l'indépendance et l'autonomie, il est donc de ce fait plus enclin à la prise de risque.

\section{Bibliographie}

Almus, M. (2002), «What characterizes a fast-growing firm», Applied Economics, vol. 34, p. 1497-1508.

Audet, J. (2003), «La veille stratégique chez les PME de haute technologie: une étude de cas par comparaisons intersites », Revue internationale PME, vol. 16, $\mathrm{n}^{\circ} 2$, p. 105-130.

Betbeze, J.-P. et C. SAINT-Étienne (2006), «Une stratégie PME pour la France», Rapport au conseil d'analyse économique, Paris, La Documentation française.

BERNASCONI, M. et F. MOREAU (2003), «L'évolution du projet des jeunes entreprises technologiques innovantes au cours des premières années: une méthode d'ap- 
préciation du cheminement stratégique», Revue internationale PME, vol. 16,

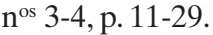

BERTHOLON, G.P. (1994), «Émergences d'entreprises, environnement local et universitaire», dans F. Bournois (dir.), Nouvelles voies pour la création et le développement d'entreprises technologiques et innovantes, Les Chemins de la Recherche $n^{\circ}$ 26, Programme Rhône, p. 35-44.

Boissin, J.-P. et M. TROMmetTER (2003), «Stratégies de croissance et contextes de gouvernement d'entreprise de biotechnologie», Revue internationale PME, vol.16, nos 3-4, p. 75-93.

CARONTINI, E. (1990), «Le rôle de l'abduction dans le processus d'interprétation (note à propos de la théorie de l'abduction chez Ch. S. Peirce) », dans L. Sfez et G. Colulée (dir.), Colloque de Cerisy, Technologies et symboliques de la communication, Grenoble, Presses universitaires de Grenoble, p. 216-227.

Chalus-SAuvannet, M.-C. (2006), «Intégration de la veille dans le système organisationnel de l'entreprise - Les enjeux pour l'innovation? », La Revue des Sciences de Gestion - Direction et Gestion, n ${ }^{\circ} 218$, p. 31-43.

Churchill, N. et V. Lewis (1983), «The five stages of small business growth», Harvard Business Review.

DAVAL, H., B. DESCHAMPS et S. GEINDRE (2002), «Proposition d'une grille de lecture des profils d'entrepreneurs », Sciences de gestion, no 32 .

DAVID, A. (1999). «Logique, épistémologie et méthodologie en sciences de gestion », VIII ${ }^{\mathrm{e}}$ Conférence de l'Association internationale de Management stratégique (AIMS), Paris, École centrale, 26-28 mai, 23 p.

DAVIDSSON, P. et J. WIKLUND (1999), «Conceptual and empirical challenges in the study of firm growth», dans D. Sexton et H. Landström (dir.), Handbook of Entrepreneurship, Malden, Mass., Blackwell Business, p. 26-44.

Delmar, F., P. DAVIDSSON et W.B. GARTNER (2003), «Arriving at the high-growth firm », Journal of Business Venturing, vol. 18, p. 189-216.

DoutriauX, G. (1994), «L'université, une pépinière d'entreprises?», dans F. Bournois (dir.), Nouvelles voies pour la création et le développement d'entreprises technologiques et innovantes, Les Chemins de la Recherche ${ }^{\circ}$ 26, Programme Rhône, p. 45-60.

Dunkelberg, W. et A. CoOper (1982), «Patterns of small business growth», Academy of Management Proceedings, p. 409-413.

ÉmIN, S. (2003), L'intention de créer une entreprise par des chercheurs publics: le cas français, Thèse de doctorat, Grenoble.

ÉMIN, S. (2006), «La création d'entreprise: une perspective attractive pour les chercheurs publics? », Revue finance, contrôle, stratégie, vol. 9, nº 3 .

FAYOLLE, A. (1994), «De l'ingénieur à l'entrepreneur: une contribution essentielle et un parcours sous influences multiples », dans F. Bournois (dir.), Nouvelles voies pour la création et le développement d'entreprises technologiques et innovantes, Les Chemins de la Recherche n 26, Programme Rhône, p. 113-138. 
GOMEZ, P.Y. (1996), Le gouvernement d'entreprise, Paris, InterÉditions.

Gulati, R., N. NOHRIA et A. ZAHEER (2000), «Strategic networks », Strategic Management Journal, vol. 21, n 3, p. 203-215.

GREINER, L.E. (1986), «Evolution and revolution as organizations grow», dans J. Williamson (dir.), The Leader Manager, John Wiley \& Sons, p. 151-164.

HAMEl, G. et C.K. Prahalad (1989), «Strategy intent», Harvard Business Review, mai-juin, p. 63-76.

HuREL, F. (2008), «Rapport à Hervé Novelli, Secrétaire d'État en charge des entreprises et du commerce extérieur, en faveur d'une meilleure reconnaissance du travail indépendant», Secrétariat d'État aux entreprises et au commerce extérieur, Paris, Ministère de l'Économie, des Finances et de l'Emploi, 51 p.

JANSSEN, F. (2005), «La conceptualisation de la croissance:l'emploi et le chiffre d'affaires sont-ils des représentations interchangeables d'un même phénomène?», Gestion 2000, vol. 22, p. 267-291.

JENSEN, M.C. et W.H. MECKLING (1976), «Theory of the firm: managerial behavior, agency cost and ownership structure », Journal of Financial Economics, vol. 3 , octobre, p. 305-360.

JULIEN, P.-A. (2001), «Les PME à forte croissance et la métaphore du jazz. Comment gérer l'improvisation de façon cohérente», Revue internationale de PME, vol.14, $\mathrm{n}^{\text {os } 3-4, \text { p. } 129-161 .}$

JULIEN, P.-A. et M. MARCHESNAY (1987), La petite entreprise, principes d'économie et de gestion, Paris, Vuibert Gestion.

Julien, P.-A. et M. MARCHESNAY (1996), L'entrepreneuriat, Paris, Economica.

Koenig, G. (1993), «Production de la connaissance et constitutions des pratiques organisationnelles », Revue de Gestion des ressources humaines, $\mathrm{n}^{\circ}$ 9, p. 4-17.

LAUFER, J. (1975), «Comment devient-on entrepreneur? », Revue française de gestion, novembre, p. 11-26.

LORRAIN, J. et L. DUSSAUlT (1988), «Les entrepreneurs artisans et opportunistes: une comparaison de leurs comportements de gestion», Revue internationale $P M E$, vol. 1, n 2, décembre, p. 157-176.

Marchesnay, M. (1998), "Confiances et logiques entrepreneuriales », Sciences de Gestion - Économies et Sociétés, numéro spécial 20e anniversaire, $\mathrm{n}^{\text {os }} 8-9$, p. 99-117.

MineR, J. B. (1990), «Entrepreneurs, high growth entrepreneurs, managers, contrasting and overlapping motivational patterns », Journal of Business Venturing, vol. $5, \mathrm{n}^{\circ} 4$, p. 221-234.

MintzBerg, H., B. Ahlstrand et J. LAmpel (1999), Safari en pays stratégie, l'exploration des grands courants de la pensée stratégique, Paris, Village Mondial.

MustaR, P. (1998), «Partnerships, configurations and dynamics in the creation and development of SMEs by researchers. A study of academic entrepreneurs in France », Industry and Higher Education, août, p. 217-221. 
PHILIPPART, P. (2008), «La création d'entreprise par les chercheurs fonctionnaires dans le cadre de la loi sur l'innovation: bilan et perspectives de recherche», $\mathrm{IX}^{\mathrm{e}}$ Congrès international francophone en entrepreneuriat et PME, Louvainla-Neuve, 28-31 octobre.

SAMMUT, S. (1996), «Processus de démarrage en petite entreprise et perspectives de croissance », III ${ }^{\mathrm{e}}$ Congrès international francophone de la PME, Université du Québec à Trois Rivières, 23-25 octobre, tome 1, p. 535-553.

SAuvé, P., A. MAhjoub et L. Pelletier (2007), «La croissance des PME: exploration des facteurs de croissance auprès d'entreprises de l'Abitibi-Témiscamingue», Ve Congrès international de l'Académie de l'entrepreneuriat, Sherbrooke, Canada, $14 \mathrm{p}$.

SCHumpeter, J.A. (1939), Business Cycles: A Theoretical, Historical, and Statistical Analysis of the Capitalistic Process, New York, McGraw-Hill.

STOREY, D. (1994), Understanding the Small Business Sector, Londres, International Thomson Business Press.

TEECE, D.J. (2007), «Explicating dynamic capabilities : the nature and microfoundations of (sustainable) enterprise performance», Strategic Management Journal, vol. 28, p. 1319-1350.

TORRÈs, O. (1998), «Vingt-cinq ans de recherche en PME: une discipline entre courants et contre-courants », dans O. Torrès (dir.), PME, de nouvelles approches, chapitre 1, Paris, Economica, 187 p.

Tushman M.L. et P. ANDERSON (1986), «Technological discontinuties and organizational environments », Administrative Science Quarterly, vol. 31, p. 439-65.

VANACKER, T. et S. MANIGART (2006), «A dynamic view on the financing of extreme growth companies», Actes de la XXVI e Conférence annuelle "Frontiers of Entrepreneurship Research », Babson College.

VARRAUT, N. (1999), «De la vision à l'intention stratégique: une application au dirigeant-propriétaire de PME», Économie et Sociétés, Sciences de Gestion, vol. 26-27, $\mathrm{n}^{\text {os }}$ 6-7, p. 146-166.

VERSTRAETE, T.(1999), Entrepreneuriat. Connaître l'entrepreneur, connaître ses actes, Paris, L'Harmattan.

VERSTRAETE, T. (2001), «Le phénomène entrepreneurial», Revue de l'Entrepreneuriat, vol. $1, \mathrm{n}^{\circ} 1$, p. 5-24.

Verstraete, T. et B. SAPORTA (2006), Création d'entreprise et entrepreneuriat, Éditions de l'ADREG, 518 p.

Weinzimmer, L.G., P.C. NySTROM et S.J. FreEMAN (1998), «Measuring organizational growth: issues, consequences and guidelines », Journal of Management, vol. 24, p. 235-262.

YIN, R.K. (1989), Case Study Research. Design and Method, Beverley Hill, Sage Foundation. 